\title{
Publisher Correction: Topological antiferromagnetic spintronics
}

Libor Šmejkal, Yuriy Mokrousov, Binghai Yan and Allan H. MacDonald

Correction to: Nature Physics https://doi.org/10.1038/s41567-018-0064-5, published online 2 March 2018.

In the version of this Review Article originally published, three of the citations corresponded to the wrong references. Ref. 16 should have corresponded to Nature 533, 513-516 (2016), ref. 17 to Nat. Mater. 16, 94-100 (2016), and ref. 18 to Phys. Rev. Appl. 6, 054001 (2016).

In addition, in Fig. 1e, the thin black data line should have been a continuation of the thick blue one; the credit citations for panels $\mathbf{b}$ and $\mathbf{c}$ of Fig. 5 were the wrong way around; they should have been to ref. 96 for $\mathbf{b}$ and ref. 93 for $\mathbf{c}$; and references 108,109 and 110 were appearing out of order, though correctly cited.

These errors have been corrected in the online version of the Review Article.

Published online: 30 May 2018

https://doi.org/10.1038/s41567-018-0171-3

\section{Publisher Correction: What's in the box?}

\section{David Abergel}

Correction to: Nature Physics https://doi.org/10.1038/s41567-018-0143-7, published online 2 May 2018.

In the version of this Research Highlight originally published, the penultimate sentence said that the absorption spectrum "does reveal detailed information about its design." It should have said that it "does not reveal detailed information about its design." This has now been corrected.

Published online: 29 May 2018

https://doi.org/10.1038/s41567-018-0185-X

\section{Publisher Correction: Synthetic antiferromagnetic spintronics}

\author{
R. A. Duine, Kyung-Jin Lee (D), Stuart S. P. Parkin (iD) and M. D. Stiles (iD)
}

Correction to: Nature Physics https://doi.org/10.1038/s41567-018-0050-y, published online 2 March 2018.

Owing to a technical error, this Perspective was originally published without its received and accepted dates in the HTML version; the dates "Received: 30 May 2017; Accepted: 17 January 2018" have now been included. The PDF is correct.

Published online: 18 June 2018

https://doi.org/10.1038/s41567-018-0195-8 